\title{
La Cirugía Mayor Ambulatoria: Rol de la Anestesia Regional
}

\author{
Ambulatory Surgery: Role of Regional Anesthesia \\ https://doi.org/10.25237/carsach2020.03
}

\author{
Dr. Alejandro Recart F. ${ }^{1}$ Dra. Andrea González S. ${ }^{1}$ \\ ${ }^{1}$ Servicio de Anestesiología, Clínica Alemana - Universidad del Desarrollo, Santiago, Chile \\ Autor corresponsal: \\ Dra. Andrea González S. \\ Servicio de Anestesiología, Clínica Alemana - Universidad del Desarrollo, Santiago, Chile \\ Dirección: Vitacura 5951, Vitacura, Santiago, Cod Postal 7650568, Chile
}

Teléfono: 56222101699

e-mail: andreagonzalezsegovia@gmail.com

ORCID iD Primer Autor: 0000-0002-5950-9560

Palabras claves: cirugía mayor ambulatoria; anestesia regional; dolor postoperatorio; bloqueo regional periférico; bloqueo fascial; anestesia libre de opioides

Key words: ambulatory/outpatient surgery; regional anesthesia; postoperative pain; peripheral block; fascial block; opioid free anesthesia

\section{Puntos claves:}

- La cirugía mayor ambulatoria es un modelo de atención sanitaria para pacientes quirúrgicos que busca que los pacientes operados completen el postoperatorio en sus domicilios, con calidad, seguridad y costo efectividad.

- Su implementación requiere la participación activa de los anestesiólogos, y en el contexto actual de retorno a la cirugía post - pandemia, es la alternativa de elección para una cirugía segura sin ocupar las camas de la institución

- La anestesia regional en todas sus formas es un componente esencial en CMA, porque mejora la calidad de atención al permitir un postoperatorio libre de dolor

\section{Resumen}

La Cirugía Mayor Ambulatoria (CMA) es un modelo de atención en salud, específico para pacientes quirúrgicos, que ha logrado un importante crecimiento en gran parte del mundo desarrollado, donde cerca del $80 \%$ de toda la cirugía electiva se realiza de esta manera. En términos generales consiste en que a determinados pacientes se les da de alta el mismo día de la cirugía y no necesitan de la cama hospitalaria para completar su proceso de recuperación. La CMA constituye una estrategia costo-efectiva comparada con la cirugía tradicional, al resolver el procedimiento quirúrgico sin comprometer la calidad de la atención ni la seguridad del paciente. Los avances en cirugía mínimamente invasiva y técnicas anestésicas que permiten una rápida recuperación, han permitido que la cirugía ambulatoria sea considerada la alternativa de elección para muchos tipos de procedimientos. Para el adecuado funcionamiento de un programa de CMA es necesario prevenir la aparición de complicaciones como el dolor postoperatorio, que puede dificultar el alta precoz de los pacientes. Para esto, la anestesia regional (AR) es muy importante y constituye un pilar fundamental para el éxito de un programa de CMA. Un adecuado control del dolor a través de la AR permite además minimizar 
el uso de opioides y sus complicaciones asociadas logrando mejores resultados para los pacientes. Gracias al apoyo de la ultrasonografía se pueden utilizar diferentes técnicas de AR, en una gran cantidad de cirugías, como bloqueos de nervio o fasciales, únicos o continuos, utilizando distintas combinaciones, dosis de anestésicos locales y drogas coadyuvantes. Para esto, resulta esencial el entrenamiento adecuado y la participación activa en todo el proceso perioperatorio de los anestesiólogos.

\begin{abstract}
Ambulatory Surgery (AS) is a specific model of health care designed for surgical patients, which has reached great evolution in the developed world, where about $80 \%$ of all elective surgery is performed this way. In general terms, these patients are discharged home the same day of surgery and do not need a hospital bed to complete their recovery process. AS is a cost-effective strategy compared to traditional care, by solving the surgical procedure without compromising quality of care or patient safety. Advances in minimally invasive surgery and anesthetic techniques that allow rapid recovery, have allowed outpatient surgery to be considered the alternative of choice for many types of surgical procedures. For the proper functioning of an AS program, it is necessary to prevent complications such as postoperative pain, that can make difficult an early and safe discharge of patients. The role of regional anesthesia (RA) is very important and is one of the fundamental pillars for the success of an AS program. Adequate pain control through RA also make possible to minimize opioid use and their associated complications and it is associated with better outcomes for patients. Due to the availability of ultrasonography, different RA techniques such as single, continuous or fascial blocks, using different combinations and doses of local anesthetics and adjunctive drugs, can be utilized in a large number of surgeries. An adequate training and active involvement of anesthesiologists in the perioperative process are essential for implementing RA for AS programs.
\end{abstract}

\title{
Introducción
}

El escenario sanitario está en continuo cambio y enfrenta enormes desafíos en el futuro cercano. Por un lado están los cambios epidemiológicos y socioeconómicos de la población, que hacen que la demanda por cirugía, y también los costos en salud, aumenten en forma sostenida ${ }^{1}$. Recientemente, la pandemia COVID 19 nos ha enfrentado a una de las situaciones más complejas de la historia reciente, lo que entre otras cosas está afectando de manera muy importante la atención quirúrgica. La no satisfacción de la demanda quirúrgica trae como consecuencia la aparición de largas listas de espera, lo cual genera gran insatisfacción en la población e incluso deterioro de su condición sanitaria. Debido a la pandemia, toda esta situación se ha hecho aún más grave alcanzando niveles de crisis sanitaria, aumentando el número de pacientes que esperan cirugía. El permanente desbalance entre la gran demanda quirúrgica y la escasa capacidad de resolución (oferta) por parte del sistema sanitario no tiene tanto que ver con la disponibilidad de pabellones, sino que más bien con la gran escasez de camas hospitalarias. A modo de ejemplo, en Chile existen 2.2 camas/ 1000 hab y el promedio OCDE es de casi 5/1000 hab ${ }^{2}$. Dado que es muy difícil y lento aumentar la oferta de camas quirúrgicas, resulta entonces imperativo mejorar la eficiencia en la utilización de los pabellones. Hoy, más que nunca, nos enfrentamos al reto de proporcionar una asistencia eficiente, de calidad y controlando los costos, por lo que estamos obligados a revisar algunas de nuestras prácticas y hacer los cambios que sean necesarios, encontrando soluciones que mejoren la atención y resuelvan los problemas de nuestros pacientes. En el escenario actual de pandemia COVID 19, cuando los diferentes centros clínicos reinician las cirugías suspendidas, la implementación de la cirugía mayor ambulatoria (CMA) aparece como una de las opciones más apropiadas para un retorno seguro a la cirugía, dado que no se necesita ocupar las camas hospitalarias, las cuales pueden ser reservadas para otro tipo de pacientes y los pacientes permanecen menos tiempo dentro del hospital, minimizando el riesgo de contagio ${ }^{3}$. Las diferentes técnicas de anestesia regional (AR) en pacientes quirúrgicos ambulatorios son un complemento esencial para asegurar un postoperatorio seguro y libre de dolor. 


\section{Metodología}

Se realizó una revisión bibliográfica no sistemática en pubmed, cochrane, google scholar con las siguientes palabras claves: ambulatory surgery; day surgery; peripheral nerve block, regional anesthesia and ambulatory surgery, outpatient surgery; role of regional anesthesia in ambulatory surgery. Se revisó estudios clínicos randomizados, estudios retrospectivos, revisiones, metaanálisis, estudios observacionales y estudios poblacionales. La selección de los artículos a incluir quedó a criterio de los autores.

\section{La Cirugía Mayor Ambulatoria}

La CMA es un modelo de gestión quirúrgica en que a los pacientes se les da de alta el mismo día de una cirugía electiva, evitando la necesidad de utilizar cama de hospitalización para poder operar y los pacientes completan su periodo de recuperación en domicilio, ofreciendo de esta manera una efectiva solución al problema de la baja oferta quirúrgica, porque se puede programar cirugías sin necesidad de ocupar camas de dotación. En la actualidad, en muchos hospitales y clínicas todavía se exige una cama para poder operar a pacientes que en la práctica son susceptibles de alta en el día ${ }^{4}$. La cama es un requisito de ingreso y las personas muchas veces no se operan no necesariamente porque no exista espacio en pabellón, sino porque no se cuenta con camas disponibles.

Los avances en cirugía mínimamente invasiva y técnicas anestésicas que permiten una rápida recuperación y libre de dolor, han permitido que la ambulatorización o cirugía de día, sea considerada la alternativa de elección para muchos tipos de procedimientos, tanto es así que actualmente en países desarrollados hasta el $80 \%$ de la cirugía electiva se

realiza de esta manera ${ }^{5}$. Comparada con la cirugía tradicional, la CMA es una estrategia costo-efectiva, al resolver los procedimientos quirúrgicos sin comprometer la calidad de la atención ni la seguridad del paciente. Este modelo de atención quirúrgica ha mostrado tener múltiples ventajas tanto clínicas (oportunidad) como administrativas (listas de espera) y financieras (ahorro de recursos). La principal son los mejores resultados para los pacientes ${ }^{6}$. Para que el modelo CMA funcione en forma eficiente, la participación del anestesiólogo es primordial, donde debe ser capaz no sólo de dominar múltiples técnicas de anestesia y analgesia, sino que de estar involucrado en todo el proceso perioperatorio de este tipo específico de pacientes quirúrgicos.

En general, en los lugares donde la CMA se realiza en forma regular, esta se lleva a cabo en unidades especiales e independientes llamadas UCA/UCMA (Unidad de CMA). Una UCMA requiere de condiciones estructurales, funcionales y también de recursos que garanticen un funcionamiento eficiente y de calidad, así como la seguridad de los pacientes. Adicionalmente, es muy importante contar con equipos de trabajo entrenados y cohesionados, que tengan protocolizada su atención clínica de manera de disminuir la variabilidad tanto de procesos como de resultados. Normalmente el ingreso hospitalario y el alta post operatoria ocurren dentro de esta misma unidad, de manera que lo ideal es que cuenten también con alguna estructura administrativa independiente. Lo habitual es que los pacientes retornan precozmente a sus actividades cotidianas luego de operarse y en general manifiestan preferencia para ser intervenidos de esta manera, porque les permite un menor tiempo de separación de su entorno, lo que es muy beneficioso especialmente en grupos de pacientes pediátricos y ancianos ${ }^{8}$. Por todo lo anterior, debe entenderse a la CMA como un modelo formal y estructurado que funciona en base a protocolos de atención clínica ${ }^{7}$. Esto es diferente a un alta precoz, en que algunos pacientes son dados de alta por sus tratantes el mismo día de la cirugía cuando han tenido un post-operatorio favorable.

Lo más destacado de la CMA es que se trata de un modelo de gestión costo-efectivo, lo cual permite disminuir transversalmente los costos en salud manteniendo la calidad de atención y la seguridad para los pacientes. Existen reportes de ahorro de costos para las instituciones que van desde un $25 \%$ hasta cerca de un $65 \%$ comparado con cirugía tradicional, solamente por el hecho de no tener que utilizar la cama hospitalaria, pero se producen además una serie de otras economías de escala al optimizar el uso de los pabellones, porque permite una mejor programación y planificación de las tablas quirúrgicas y los convierte en la práctica en pabellones de alto rendimiento 5,9 . Evidentemente la cama no utilizada por los pacientes quirúrgicos se re -destina a un uso alternativo, lo que agrega mayor valor al mod- 
elo. Esta ha sido la causa de su implementación definitiva en muchos lugares, independientemente de si se trata de salud pública o privada.

La CMA si bien va en aumento, no es una práctica habitual o consistente en nuestro medio y al comparar con lo que actualmente ocurre en otros países, donde se observa que pacientes intervenidos del mismo tipo de cirugía son dados de alta en el día y nosotros los mantenemos hospitalizados por uno, dos o más días. Uno de los argumentos para no iniciar programas de este tipo tiene que ver con los eventuales riesgos o complicaciones que podrían ocurrir al regresar los pacientes a su casa el mismo día de la cirugía. Existe numerosa evidencia que demuestra que, cuando la CMA se realiza de la manera apropiada, siguiendo en forma estricta las guías, protocolos y recomendaciones al respecto, no existe diferencia entre ésta y la cirugía con hospitalización en lo que tiene que ver con la seguridad de los pacientes ${ }^{10}$. Si bien la incidencia de complicaciones graves relacionadas a la CMA es muy baja, es posible que aparezca dolor, náusea, somnolencia y otras situaciones que no suelen poner en riesgo a los pacientes, pero si pueden retrasar o impedir el alta.

No existen buenas razones que puedan explicar por qué la CMA no es un estándar de cuidado entre nosotros, cuando existe numerosa experiencia y literatura que la respalda. Si bien a nivel nacional existen centros donde la CMA está más desarrollada, no es todavía una práctica consistente. Este tipo de iniciativas significa en la práctica que las instituciones y las personas (pacientes, profesionales) deban hacer un cambio a sus prácticas habituales, y lo usual es que el cambio genere resistencia, en este caso a la manera en que siempre se ha realizado la cirugía. A nivel público puede ser que existan restricciones presupuestarias para construir o equipar una unidad de CMA, o bien que en algunos servicios existan incentivos para mantener a los pacientes hospitalizados para no perder su número habitual de camas asignadas $^{4}$. A nivel privado lo más frecuente de observar es la reticencia al alta en el día porque el asegurador (Isapre) no va a reembolsar (o puede reembolsar menos) una prestación ambulatoria versus hospitalizada. En nuestra experiencia, con miles de pacientes operados dentro de un programa de CMA, no se ha presentado un caso de este tipo, porque el reembolso tiene que ver más bien con la complejidad de la cirugía o guarismo que con el hecho de si pasa la noche o no dentro de la Institución. Por lo tanto, no hemos observado esta situación porque la gran mayoría de las cirugías ambulatorias tiene un nivel de complejidad tal, que la Isapre reembolsa igual como si fuera hospitalizado. Sin embargo, esta situación sigue generando confusión y debiera ser resuelta a la brevedad.

La CMA no se trata de una técnica quirúrgica o anestésica específica, sino que consiste en organizar la asistencia del paciente quirúrgico de modo que éste se encuentre en su domicilio el mismo día de la operación y con las mismas garantías que si estuviera hospitalizado. La prevención y el manejo de las complicaciones, especialmente el control del dolor postoperatorio serán vitales para que el paciente pueda realizar un postoperatorio seguro y retomar a la brevedad sus actividades habituales.

\section{Implementación de la Cirugía Mayor Ambulatoria}

Una gran variedad de cirugías electivas pueden hacerse de manera ambulatoria y se pueden utilizar distintas técnicas quirúrgicas, anestésicas y analgésicas ${ }^{4,6}$. Cada lugar deberá determinar cuáles cirugías podrán ser ambulatorizadas, considerando:

- Minimizar el trauma quirúrgico, prefiriendo técnicas mínimamente invasivas.

- Preferir cirujanos y anestesiólogos más experimentados de manera de disminuir los tiempos quirúrgicos y la posibilidad de complicaciones así como también hacer más expedito el proceso de recuperación y alta.

- El dolor postoperatorio debe poder ser controlado con analgesia oral y/o con técnicas de bloqueos anestésicos regionales.

- Elegir cirugías de menos de 120 min de duración.

- Seleccionar aquellas cirugías con baja probabilidad de sangrado post operatorio.

En nuestra experiencia con el programa de CMA de Clínica Alemana, contamos en la actualidad con más de 140 códigos quirúrgicos diferentes incorporados, de todas las especialidades quirúrgicas, desde cirugía infantil, plástica, digestiva, vascular y especialmente traumatología (tabla1), donde gracias al apoyo de diferentes técnicas de anestesia 
regional, incluso pacientes de cirugías más complejas como artroscopia de hombro, logran ser dados de alta en el día y completar un postoperatorio satisfactorio.

Una de las claves de los buenos resultados de nuestro programa de CMA es que el proceso de atención clínica está escrito y es conocido por todos quienes participen del cuidado de estos pacientes y además está continuamente siendo sometido a evaluación y mejora continua. Existe una etapa de evaluación previa donde a los pacientes se les selecciona y evalúa antes de la operación, se les educa en relación a su cirugía (incluyendo indicaciones preoperatorias) y a su postoperatorio en domicilio. Esto permite una mejor adaptación y adherencia a todo su perioperatorio y evita cancelaciones de procedimientos. Luego de operarse, y cuando cumplen determinados criterios de recuperación, son dados de alta pero continúan bajo vigilancia por nuestro mismo equipo clínico, realizándose un seguimiento postoperatorio vía telefónica y dejando además un teléfono abierto las 24 horas en caso de que algún paciente necesitara comunicarse directamente. Se cuenta además con normas de actuación en caso que el paciente presente alguna complicación u otra situación médica en domicilio.

No todos los pacientes son candidatos a operarse en forma ambulatoria ni toda cirugía es posible de ser realizada bajo esta modalidad. Un adecuado programa de CMA debe tomar en cuenta las comorbilidades y las características socioculturales de los pacientes en los criterios de inclusión y exclusión, además siempre es necesario conocer las características del centro quirúrgico y la experiencia del equipo tratante. En general se prefiere ofrecer CMA a pacientes sanos o con sus comorbilidades controladas (ASA I o II) y que cuenten con un adulto responsable en domicilio para las primeras 24 horas. Se sugiere evitar incluir pacientes de edades extremas (menores de 6 meses o mayores de 85 años); obesos mórbidos (IMC > 35), que no cuenten con un adulto responsable en domicilio o vivan a más de una hora de la clínica u hospital. Sin embargo la última palabra siempre es del equipo clínico: si luego de la evaluación, se decide que determinado paciente debe ser resuelto con hospitalización, entonces sale del programa. En CMA la calidad de atención no radica en si se hospitaliza o no, sino en la calidad de todo el proceso perioperatorio.

La experiencia en diversos países indica que la CMA es muy bien aceptada tanto por los clínicos como por la comunidad una vez que todos comprenden sus beneficios y también sus limitaciones. Una adecuada estrategia de implementación debe incluir una aproximación gradual, con los cirujanos y anestesiólogos interesados, pero también integrar al personal de enfermería y a todo el equipo de salud, que son claves para efectivamente implementar este tipo de cambios. En el modelo CMA es fundamental un enfoque multidisciplinario y trabajo en equipo.

El rol de cada miembro del equipo es importante: los cirujanos requieren experiencia, para minimizar el trauma propio de la cirugía, las complicaciones y acortar los tiempos quirúrgicos. Además, es necesario que los pacientes conozcan desde el principio que serán operados sin hospitalización, y esa información debe venir primero desde los tratantes.

El anestesiólogo deberá ser capaz de, independiente de la técnica usada, permitir a los pacientes recuperar a la brevedad el estado previo a la operación, libre de dolor, náuseas y otras complicaciones para así facilitar el flujo de los pacientes a través de todo el perioperatorio. Esto es lo que se ha llamado anestesia fast-track o de rápido recambio.

El personal de enfermería es fundamental. Participan de la selección y educación de los pacientes y en el seguimiento postoperatorio, dándole continuidad a la atención clínica.

Inicialmente se deberían incorporar pacientes y procedimientos en los que todos estén confortables respecto a su ambulatorización, para luego ir incorporando tanto pacientes como cirugías más complejas. Cada unidad va aprendiendo de su propia experiencia y lo habitual es que cuando este modelo está transversalmente aceptado y probado su eficacia, se integran procedimientos e incluso cirujanos, que inicialmente no participaban. Esto es exactamente lo que hemos observado en nuestra institución.

Uno de los elementos más importantes que explican el éxito y la masividad de la CMA internacionalmente es que se generan incentivos positivos para todos quienes participan del proceso de atención: Las instituciones y las aseguradoras logran mayor eficiencia y control de costos; los cirujanos pueden operar más y con mejor predictibilidad a la hora de agendar, pero por sobre todo los pacientes, que si son del mundo público obtienen oportunidad de atención y los privados menores copagos y ahorro de costos en general ${ }^{8,9}$.

Para medir la eficiencia, calidad y seguridad de un programa de CMA se utilizan indicadores clínicos como el Î́ndice de Reingresos que es el paciente que fue dado de alta y regresó posteriormente al hospital, (ya sea al servicio de urgencia o directamente a re hospitalizarse) o el Índice de Hospitalización: paciente que no pudo ser dado de alta a 
pesar de que estaba programado para ello. Es frecuente que el alta se retrase o incluso que un paciente pueda tener que re-hospitalizarse cuando no se logran prevenir adecuadamente la aparición de las complicaciones, sobre todo el dolor. En este punto el rol del anestesiólogo cobra particular relevancia y es fundamental para el éxito de estos programas.

La evidencia está mostrando que la CMA seguirá en expansión. La continua incorporación de nueva tecnología, con cirugías cada vez menos invasivas y la creciente necesidad de controlar el aumento de los costos en salud hacen probable que esta estrategia se convertirá en la alternativa principal para los pacientes quirúrgicos electivos y pronto será necesario explicar, tanto a los pacientes, instituciones y aseguradoras, el por qué el paciente tuvo que ser hospitalizado luego de su cirugía, en vez de ser dado de alta en el día ${ }^{12}$.

\section{Rol de la Anestesia Regional en CMA}

Un programa de CMA debe contar de manera prioritaria con estrategias para el control del dolor postoperatorio. La presencia de esta complicación es uno de los motivos principales de reingresos e insatisfacción de los pacientes y adicionalmente, un adecuado control del dolor permite ambulatorizar cirugías que de otra manera necesitan hospitalización exclusivamente para la administración de analgesia. Lo habitual es implementar técnicas de analgesia preventiva y multimodal y, por sobre todo, utilizar diferentes técnicas de AR cuando esto sea posible.

Tradicionalmente la AR se ha realizado en cirugías traumatológicas, ya que presentan dolor moderado a severo, que es difícil de controlar con analgésicos endovenosos u orales. Además, si la cirugía traumatológica se realiza en forma ambulatoria, donde un $20-40 \%$ de los pacientes presentan dolor moderado a severo ${ }^{13}$, más aún es recomendable realizar bloqueos de nervio periférico (BNP) para lograr alta dentro del día. Cuando los pacientes reciben un BNP, se ha visto que más del $94 \%$ de ellos tiene cero o leve dolor en el postoperatorio inmediato y que la intensidad del dolor a las 48 y a las 72 horas es menor comparado con los pacientes que no reciben $\mathrm{BNP}^{14}$. En la literatura hay diferentes reportes sobre la intensidad del dolor y el consumo de opioides postoperatorio después de las 24 horas cuando se realiza BNP, esto depende de los anestésicos locales (AL) utilizados, si son de corta o larga duración, del uso de coadyuvantes y si es un bloqueo único o continuo. Mientras más tiempo dure un BNP, mayor será el beneficio en la disminución del consumo de opioides. Además los BNP pueden disminuir la incidencia de dolor crónico postquirúrgico que en el contexto de cirugía traumatológica puede llegar hasta el 50\%. ${ }^{15,16,36}$

El uso excesivo de opioides para el control del dolor postoperatorio en CMA favorece la aparición de complicaciones como náuseas, vómitos (NVPO) y somnolencia, las que también retrasan el alta y generan incomodidad a los pacientes en sus domicilios. A pesar de la profilaxis y tratamiento, la incidencia de NVPO en CMA es alta (20-40\%), además un $1 / 3$ de los pacientes presentan NVPO después del alta que puede durar hasta 5 días, siendo esto de difícil manejo 17,18

La incidencia de NVPO en CMA traumatológica puede llegar hasta un $62 \%$ cuando solo se realiza bajo anestesia general, pero cuando se realiza exclusivamente bajo AR la incidencia puede llegar a un 5-12\%, pero si la AR se combina con anestesia general este beneficio disminuye. ${ }^{13}$ La utilidad de las técnicas regionales en la prevención de NVPO va más allá del periodo postoperatorio inmediato, porque consigue disminuir la utilización de opioides en el domicilio y las NVPO después de alta, evitando la re-hospitalización por estas causas. ${ }^{17,18}$

La disminución del uso total de opioides que consigue la AR, tiene un efecto positivo no solo en la disminución de complicaciones, sino que además consigue un beneficio desde el punto de salud pública: En EE.UU existe una crisis de consumo y abuso de los opiáceos, la cual se ha generado en parte por la indicación excesiva de opiáceos en el postoperatorio lo que favorece el consumo recreativo. El 2017 en ese país hubo más de 17000 muertes por sobredosis de opiáceos relacionadas con la prescripción de estos medicamentos para manejo del dolor. ${ }^{19}$ Esto podría disminuir utilizando en forma adecuada las diferentes técnicas regionales para analgesia postoperatoria. Si bien en nuestra realidad nacional no hay disponibilidad de opiáceos orales y estamos lejos de una crisis de abuso como la descrita, es fundamental prevenir y no crear la necesidad de incorporarlos en nuestro arsenal nacional de medicamentos.

Otra ventaja de la AR es que ha permitido que pacientes ASA 3 puedan ser sometidos a CMA, cuando la cirugía se realiza exclusivamente bajo esta técnica, porque presenta un menor riesgo de complicaciones, mayor estabilidad 
hemodinámica, y finalmente permite la ambulatorización en estos pacientes, lo cual de otra manera no sería posible. $^{20}$

La evidencia ha demostrado que la AR en CMA consigue una alta satisfacción de los pacientes $(97 \%)$ dado que se logra un adecuado manejo del dolor, sin efectos secundarios de los analgésicos opioides. Mientras más efectivos y de efecto prolongado sean los bloqueos, los pacientes presentan una mayor satisfacción usuaria en comparación con las cirugías realizadas solo con anestesia general o bloqueos fallidos o de corta duración. ${ }^{13,21}$.

En CMA, la AR ha logrado disminuir los costos, las readmisiones, la carga asistencial de enfermería en la recuperación y/o en la unidad de hospitalización transitoria e incluso puede lograr hacer bypass de la unidad de recuperación, trasladándolos directamente a la unidad transitoria de hospitalización o prealta ${ }^{22}$, que en este periodo de pandemia es altamente deseable para disminuir la cercanía y acumulación de pacientes en áreas comunes como la recuperación.

Williams y Kentor analizaron 948 pacientes sometidos a reparación de LCA en forma ambulatoria con anestesia general (con y sin bloqueo de nervio periférico) versus anestesia raquídea (con y sin bloqueo de nervio periférico) y concluyeron que los pacientes que recibieron solo técnica regional lograban en un mayor porcentaje de by pass a la unidad de recuperación comparado con los pacientes que solo recibieron anestesia general. El uso de bloqueo de nervio periférico redujo la admisión a la unidad de recuperación en un $18 \%$, logrando disminuir un $12 \%$ el costo hos-

pitalario y la admisión hospitalaria disminuyó un 13\%, logrando una reducción de costo hospitalario de un $11 \%{ }^{22}$ En suma, la AR en CMA, al presentar los beneficios ya descritos, puede disminuir los costos hospitalarios hasta en un $23 \%$, sin embargo esta reducción dependerá de la realidad local, de la organización de cada institución, de la posibilidad de realizar estas técnicas de acuerdo a la disponibilidad de recursos y experiencia de los anestesiólogos, ya que cuando falla la AR, los costos son más altos que cuando se realiza solo anestesia general. ${ }^{22,23}$

\section{Aporte de la anestesia regional bajo ecografía a la CMA}

La aparición de la ecografía aplicada en AR junto con el mayor conocimiento anatómico han permitido desarrollar técnicas regionales principalmente sensitivas, que solo se pueden realizar bajo ecografía, como por ejemplo el bloqueo del canal del aductor e IPACK que logran la deambulación y kinesioterapia precoz con un buen control del dolor en cirugía mayor de rodilla ${ }^{24,25}$ Existe experiencia a nivel local de cirugías de reparación del ligamento cruzado anterior y de reemplazo articular de rodilla en forma ambulatoria, en pacientes seleccionados y cuando se realizan bajo un adecuado protocolo de CMA apoyado con estas técnicas de AR.

Además la ecografía ha permitido nuevos bloqueos más allá de la cirugía traumatológica, que no abordan directamente un nervio o plexo si no que son bloqueos que van entre planos musculares llamados fasciales. ${ }^{20,24} 34$ Estos bloqueos, en teoría, no tendrían riesgo de daño neural como los BNP. Se ha demostrado que disminuyen el consumo de opiáceos postoperatorio en cirugías de tronco como: colecistectomía laparoscópica, hernias, cirugía de mama entre $\operatorname{otras}^{34}$.

\section{Implementación de la Anestesia Regional en la CMA}

$\mathrm{Al}$ tratar de convertir un centro ambulatorio en uno orientado en anestesia regional es fundamental que todo el equipo y personal de salud no sólo esté familiarizado con las técnicas regionales a realizar, sino que también entienda la importancia y la necesidad de incorporarlas a este modelo para mejorar la calidad de atención del paciente. ${ }^{21} \mathrm{La}$ mayor barrera para incorporar la AR en CMA tiene que ver con la inercia y la resistencia natural al cambio por parte del equipo médico, ya que esto implica trabajo en equipo y mayor coordinación entre anestesiólogos y entre estos y el resto del equipo quirúrgico, objetivo que no siempre es fácil de alcanzar, por ende el anestesiólogo con más experiencia en AR que lidere esta implementación, debe poseer habilidades blandas para lograr una comunicación efectiva con todos los integrantes involucrados en este cambio de funcionamiento 27

Es necesario señalar que la AR en CMA debe ser realizada inicialmente por anestesiólogos que ya están familiarizados con los BNP o bloqueos fasciales más comunes descritos en la literatura, los cuales deben ser realizados prefer- 
entemente previo a la cirugía, idealmente en el menor tiempo posible, pero con alta tasa de éxito, baja tasa de complicaciones y/o falla del bloqueo, para así lograr el alta segura del paciente el mismo día de la operación. Cuando esto se logra, los cirujanos se convierten en los mejores aliados y después son ellos quienes solicitan que les realicen bloqueos a sus pacientes, ya que han visto las ventajas y la evolución en el postoperatorio. Aprovechando este entusiasmo, se les debería solicitar que en la consulta pre quirúrgica les expliquen a los pacientes que serán sometidos a alguna técnica de AR por parte del anestesiólogo, así, la aceptación por parte del paciente será mayor. ${ }^{26,27}$

Con el objetivo de mejorar la eficiencia de los pabellones, idealmente, los BNP se deberían realizar en un lugar diferente al pabellón donde se realizará la cirugía, mientras se realiza la cirugía previa, de manera de funcionar en forma paralela; puede ser en la recuperación o en un pabellón desocupado o una sala contigua que muchas veces es llamada sala de bloqueo o de preanestesia. Lo importante es que debe realizarse en un lugar cercano a los pabellones, con la implementación recomendada para el uso de anestesia regional: monitor, carro de insumos para realizar bloqueos periféricos, ecógrafo, kit de lípidos y carro de reanimación cercanos ante cualquier emergencia. ${ }^{26,28,35}$ Además debería contar con un técnico de anestesia a cargo de la sala y una enfermera presente previo al bloqueo para realizar la pausa de seguridad y así confirmar lateralidad y evitar errores. ${ }^{26,29}$

El funcionamiento en forma paralela en el proceso quirúrgico ha demostrado disminuir los tiempos de anestesia y el tiempo de recambio de pacientes, que en el caso de CMA es esencial debido al limitado horario de funcionamiento en el día y que eventualmente permitiría realizar 1,7 más cirugías considerando tiempos quirúrgicos cortos y con un horario de funcionamiento de 8 horas. ${ }^{30}$ Además, el funcionamiento en forma paralela tiene otras ventajas:

1) Permite realizar el bloqueo previo a la cirugía, disminuyendo los requerimientos anestésicos intraoperatorio, evitando uso elevado de opiáceos y previene el dolor crónico postoperatorio. Se ha demostrado que cuando el bloqueo se realiza después de la cirugía, se pierde el efecto preventivo de los BNP en la sensibilización del sistema nervioso central y por ende no logra prevenir la prevalencia de dolor crónico postoperatorio. ${ }^{15,16}$

2) Permite el tiempo necesario para que el bloqueo sea efectivo y si es insuficiente, se pueda complementar.

3) Permite tener la tranquilidad y el tiempo necesario para enseñar a becados o subespecialistas u a otros anestesiólogos que recién están aprendiendo las técnicas regionales.

4) Permite tener el tiempo necesario para realizar trabajos de investigación en técnicas de AR que requieren de evaluación post BNP y previo a la cirugía.

El tener un espacio físico exclusivo para realizar los BNP todos los días disponibles no es una condición fundamental para incorporar la AR en CMA, también como se mencionó, se podría utilizar la recuperación o un pabellón contiguo desocupado, lo importante es intentar realizar procesos paralelos en CMA, por ejemplo, aprovechar el tiempo de aseo de un pabellón para realizar AR en un lugar cercano a pabellón, pero para que se lleve a cabo en forma eficiente, se requiere una gran proactividad del anestesiólogo.

Si se quiere implementar una sala de bloqueo se debe realizar un análisis de costo-beneficio, considerando la cantidad de pabellones disponibles, si hay o no bloques de horarios de equipo quirúrgicos en las cuales las cirugías se benefician de la AR, si existe o no la disponibilidad del personal en horario continuo específico para esta sala y si no existe se deberá determinar si es costo-eficiente contratar personal para implementarla, por lo tanto, cada centro deberá evaluar sus posibilidades para aprovechar al máximo los beneficios de la $\mathrm{AR}$ en los pacientes quirúrgicos ambulatorios.

$\mathrm{Al}$ incorporar una sala de bloqueo, ésta se puede organizar en 2 formas distintas de funcionamiento:

1 Un anestesiólogo inicia proceso anestésico en sala de bloqueo y continua con el paciente en pabellón, mientras otro anestesiólogo continúa con el siguiente caso en la sala de bloqueo

2 Tener un equipo de bloqueo donde los anestesiólogos con mayor experiencia en AR son los que realizan todos los bloqueos en esta sala y otro anestesiólogo se encarga de la anestesia general o de sedación en el pabellón.

Chin y colaboradores realizaron un análisis pre y post implementación de un equipo de anestesiólogos regionalistas y demostró que cuando los bloqueos eran realizados por el equipo eran más efectivos, tenían mayor tasa de éxito y 
los pacientes se sentían más satisfechos, evidenciando que es mejor tener pocos anestesiólogos entrenados realizando todos los bloqueos que todos los anestesiólogos realizando unos pocos bloqueos. Esto mismo ocurre con el asistente o auxiliar de anestesia quien al ser uno o pocos, aumenta su conocimiento sobre los procedimientos de AR, ya que están familiarizados con las técnicas, mejorando la eficiencia, seguridad y calidad del procedimiento. ${ }^{31}$

$\mathrm{Al}$ implementar un equipo de bloqueo en el centro ambulatorio, éste debe contemplar la existencia de un consentimiento informado de BNP que sea específico y distinto del consentimiento de la anestesia, ya que serán 2 anestesiólogos diferentes quienes realizarían los procedimientos anestésicos.

Las técnicas de AR en CMA idealmente deberían ser implementadas no sólo en cirugías traumatológicas, sino que también en las cirugías de tronco, donde la evidencia ha demostrado un beneficio en la incorporación de la AR en el manejo del dolor, como: cirugía menor de mama, cirugía de hernias inguinales (abierta o laparoscópica), colecistectomía laparoscópica entre otras. ${ }^{34}$ En la tabla 2 se describen las cirugías ambulatorias en las cuales la AR ha demostrado utilidad en el manejo del dolor postoperatorio.

En relación a cual AL utilizar (si es de corta o larga duración), si se usa coadyuvante o si se realiza BNP continuo, dependerá de la intensidad del dolor que producen las cirugías en el postoperatorio y de la disponibilidad de recursos de cada centro ambulatorio. Es fundamental que esté estandarizado de acuerdo a la evidencia y que los anestesiólogos que realicen las técnicas regionales tengan claridad en el protocolo acordado, para así evitar readmisiones por dolor de rebote al desaparecer el efecto del bloqueo en cirugías conocidas por dolor moderado/severo por varias horas o días. ${ }^{32,36} \mathrm{La}$ incorporación de técnicas regionales continuas para manejo del dolor domiciliario depende no sólo del nivel de experiencia del grupo de anestesiólogos, sino también de la posibilidad de contar con un servicio de dolor que permita el control de los pacientes en forma ambulatoria que esté disponible 24/7 para resolver cualquier complicación o problema que se pudiera presentar en el domicilio y que requiera revisión por un anestesiólogo. ${ }^{27}$

Por último es importante definir con el equipo de enfermería de pabellón la utilización de algún score de by-pass de la recuperación. Existen diferentes scores descritos en la literatura para estos efectos. Uno de ellos es el de Williams y colaboradores, que incluye evaluar un total de 8 items, pero que no incluye dolor, NVPO, prurito o escalofríos, ya que considera tolerancia cero a la presencia de estas complicaciones. ${ }^{33}$ Este score junto con la tolerancia cero ha ayudado a la derivación de los pacientes a la unidad de pre alta, maximizando de esta manera el uso de la recuperación y las ventajas de la AR. $22,27,33$

\section{Conclusión}

En conclusión la CMA es el modelo de gestión al cual las instituciones de salud deberían aspirar e implementar para satisfacer parte de la alta demanda quirúrgica de manera organizada, segura, eficiente y con contención de costos. El rol del anestesiólogo es facilitar y liderar este cambio, ayudando también a promover la incorporación de técnicas de anestesia y analgesia regional, lo que contribuye a disminuir aún más los costos, mejorando los resultados perioperatorios y lo más importante, con una alta satisfacción usuaria. En el futuro, la mayor parte de la cirugía electiva se va a resolver dentro de un modelo de CMA, sobre todo gracias a la incorporación de las diferentes posibilidades que ofrece la AR.

\section{Bibliografía:}

1.- Porter M, Kaplan R.: How to Solve the Cost Crisis in Health Care. HBR. Dec 2011

2.- https://www.oecd.org/els/health-systems/Briefing-Note-CHILE-2014-in-Spanish.pdf

3.- American Society of Anesthesiologists. Roadmap for Resuming Elective Surgery after COVID-19 Pandemic. https://www.asahq.org/about-asa/newsroom/news-releases/2020/04/joint-statement-on-elective-surgery-aftercovid-19-pandemic 
4.- Recart, A.: Cirugía Mayor Ambulatoria. Una nueva forma de entender la medicina quirúrgica. Revista Médica Clínica Las Condes, 2017;28(5): 649-812

5.- Castoro C. Policy Brief Day Surgery: Making it Happen. IASS, 2007 www.euro.who.int/document/e90295

6.- White PF, Recart A. : Ambulatory (Outpatient) Anesthesia en Miller's Anesthesia, 6th ed.Copyright (C) 2005 Churchill Livingstone, ( Elsevier) Chapter 68.

7.- Manual Unidad de Cirugía Mayor Ambulatoria, Estándares y recomendaciones. Ministerio de Sanidad y Consumo España. www.msc.es/organizacion/sns/planCalidadSNS/docs/guiaCMA.pdf

8.- Jarrett P, Staniszewski A. The development of ambulatory surgery and future challenges. In: Lemos P, Jarrett PEM, Philip B (eds). Day surgery - development and practice. London: International Association for Ambulatory Surgery, 2006: 89-124.

9.- Joshi GP Efficiency in ambulatory surgery center. Current Opinion in Anaesthesiology 2008, 21:695-698.

10.- Shnaider I, Chung F. Outcomes in day surgery. Curr Opin Anaesthesiol. 2006 Dec;19(6):622-9.

11.- Merril D. : Management of outcomes in the ambulatory surgery center: the role of standard work and evidencebased medicine. Curr Opin Anaesthesiol. 2008;21:743-7.

12.- ASCs: A Positive Trend in Health Care. https://www.ascassociation.org/advancingsurgicalcare/aboutascs/industryoverview/apositivetrendinhealthcare

13.- Jacob A, Walsh M, Dilger J, Role of regional anesthesia in the ambulatory environment, Anesthesiol Clin 2010; 28:251-266

14.- Malchow R, Gupta R, Comprehensive Analysis of 13897 consecutive Regional anesthetics at an Ambulatory Surgery Center, Pain Medicine 2017; 0:1-17

15.- Rivat C, Bollag L, Richebé P, Mechanisms of regional anaesthesia protection against hyperalgesia and pain chronicization, Curr Opin Anaesthesiol, 2013; 26 (5):621-25

16.- Pedersen J, Crawford M, Dahl J et col, Effect of preemptive nerve block on inflammation and hyperalgesia after human thermal injury, Anesthesiology, 1996;84:1020-1026

17.- Geralemou S. Gan T.J, Assessing the value of risk indices of postoperative nauseas and vomiting in ambulatory surgical patients Curr Opin Anesthesiol, 2016; 29(6): 668 - 72

18.- Rawal N. Postdischarge complications and rehabilitation after ambulatory surgery, Curr Opin Anasthesiol 2008;21:736-742

19.- Zaveri S, Nobel T, Khetan P, The opiod give and take: the effect of overprescribing on patient opioid consumption patterns following ambulatory Surgery. The American Surgeon 2020;86 (5):549-550

20.- Ardon A, Prasad A, Regional anesthesia for ambulatory anesthesiologists, Anesthesiology Clin 2019;37(2):265-287

21.- Teunkens A, Vanhaecht K, Vermeulen K, Fieuws S, Measuring satisfaction and anesthesia related outcomes in a surgical daycare center: a three-year single-center observational study, Journal of Clinical Anesthesia,2017;43:15-23

22.- Williams B, Kentor M, Economic of nerve block pain management after anterior cruciate ligament reconstruction, anesthesiology 2004; 100:697-706

23.- Schuster et al, Cost drivers in anesthesia manpower, technique and other factors, Curr Opin Anaesthesiol, 2006; 19(2):177-84

24.- Li J, Lam D, King H, Novel regional anesthesia for outpatient surgery, curr pain head reports 2019, 23:69

25.- Kim D, Bajeathe J, Lin Y et al, Addition of Infiltration Between the popliteal artery and the capsule of the posterior knee and adductor canal block to periarticular injection enhances postoperative pain control in total knee arthroplasty: A Randomized Controlled Trial, Anesth Analg, 2019;129(2):526- 535 
26.- Ludwin, D, Setting up an ambulatory regional anesthesia program for orthopedic surgery, Anesthesiology Clin 2014; 32:911-921

27.- Williams B, Kentor M Making an ambulatory surgery center suitable for regional anaesthesia, Best Practice research Clinical Anesthesiology 2002; 16(2) 175-194

28.- Faddoul a, Bonnet F, Is there a place for regional anesthesia in nonoperating room anesthesia? Curr Opin Anaesthesiol, 2020;33(4):561-565

29.- McLellan EJ, Hade AD, Plecanos A et al, Introduction of a mandatory pre-block safety checklist into a regional anesthesia block room service: a quality improvement project. Anaesth Intensive Care. 2018:46(5):504-509

30.- El-BighdadlyK, Nair G, Pawa A, Impact of parallel processing of regional anesthesia with block rooms on resource utilization and clinical outcomes: a systematic review and meta-analysis. Reg Anesth Pain Med, 2020;45(9):720-726

31.- Chin a, Heywood L, Lu P et al, The effectiveness of regional anaesthesia before and after the introduction of a dedicated regional anaesthesia service incorporating a block room, Anaesth Intensive Care 2017;45(6):714-719

32.- Dada O, González Z.A Does Rebound pain after peripheral nerve block for orthopedic surgery impact postoperative analgesia and opioid consumption? A narrative Review, Int J. Environ. Res Public Health, 2019; 16: 3257

33.- Williams B, Kentor M, Williams J, Pacu Bypass after outpatient knee surgery is associated with fewer unplanned Hospital admissions but more phase II nursing Interventions, Anesthesiology, 2002; 97(4):981-988

34.- Chin K, Mcdonnell J, Carvalho B et cols, Essentials of our current urderstanding: abdominal wall blocks. Reg Anesth Pain Med, 2017;42(2):133-83

35.- Brown B, Khemani E, Lin C et al, Improving patient flow in a regional anesthesia block room, BMJ Open Qual 2019;8(2)

36.- Rana M, Desai R, Tran L, Perioperative pain control in the ambulatory setting, Curr Pain Headache Rep 2016; 20(3): 18

37.- Hamilton G, Ramlogan R, Lui A, Peripheral nerve blocks for ambulatory Shoulder surgery, Anesthesioloy 2019; 131:1254-63

38.- Saporito A, Anselmi L, Sturini E, et col, Is outpatient continuous regional analgesia more effective and equally safe than single shot peripheral nerve blocks after ambulatory orthopedic surgery? Minerva Anesthesiologica 2017;83(9):972-981

39.- Yadeau J, Fields K, Khan R, et cols, Readiness for discharge after foot and ankle surgery using peripheral nerve blocks:A randomized controlled trial comparing spinal and general anesthesia as supplements to nerve blocks, Anesth analg 2018;127(3):759-766

40.- Chan V, Peng P, Kaszas Z et cols, A comparative study of general anesthesia, intravenous regional anesthesia and axillary block for outpatient hand surgery: clinical outcome and cost analysis. Anesth analg 2001;93:1181-1184

Tabla 1: Principales cirugías ambulatorias por especialidad 


\begin{tabular}{|c|c|c|}
\hline & & Síndrome Túnel del Carpo \\
\hline & Mano & Fractura de Escafoides \\
\hline & & Tendovaginosis de Quervain \\
\hline & & Fractura de Clavícula \\
\hline & (7) & Manguito Rotador \\
\hline & & Disfunción patelo-femoral \\
\hline & Rodilla & Menisectomia artroscópica \\
\hline & & Reparación Ligamento Cruzado Anterior \\
\hline Iraumatologia & & Rotura Tendón de Aquiles \\
\hline & Iopilio y Ple & Hallux Valgus \\
\hline & Cadera & Artroscopía de cadera \\
\hline & Columna & Hernia núcleo pulposo simple \\
\hline & & Hernia umbilical \\
\hline & Cirugía Digestiva & Colecistectomía \\
\hline & & Hemorroidectomía \\
\hline & & Safenectomía \\
\hline & vasculal & Fístulas arterio-venosas \\
\hline & Cabeza y Cuello & Cirugía micrográfica \\
\hline & & Tumores piel \\
\hline Cirugía & Mama & Tumorectomía. \\
\hline & & Mastectomía parcial \\
\hline & & Orquiectomía \\
\hline & काषugia & Vasectomía \\
\hline & Oído & Timpanoplastía \\
\hline & Naríz & Septoplastía \\
\hline Otorrinolaringología & & Turbinectomía \\
\hline & Faringe & Adenoamigdalectomía \\
\hline Cirugía Plástica & Estética & Mastopexia con implantes \\
\hline Oftalmología & Cámara anterior & Cataratas \\
\hline Cirugía Infantíl & Genital & Fimosis \\
\hline
\end{tabular}

Tabla 2: Cirugías mayor ambulatorias más frecuentes y bloqueos de plexo, nervio o interfasciales descritos en cada cirugía que ayudan al manejo del dolor postoperatorio.

\section{Cirugía Mayor Ambulatoria}

Cirugía de mama

Cirugía de Hernia umbilical
Bloqueo de Plexo, Nervio periférico o Fascial

BPV, PECS, ESP, Serrato anterior,

Bloqueo Vaina de los rectos, TAP 
Cirugía de Hernia Inguinal

Colecistectomía laparoscópica

Histerectomía laparoscópica

Cirugía de fractura de clavícula

Cirugía artroscópica de hombro

Cirugía de fractura de extremidad superior

Cirugía menor de mano

Cirugía artroscópica de cadera

Cirugía artroscópica de rodilla

Cirugía Reparación de ligamento cruzado Anterior

Cirugía de tobillo y pie
$B P V$, Bloqueo llioinguinal, TAP

BPV, TAP block, Cuadrado lumbar

TAP block

Bloqueo de plexo cervical, Bloqueo interescalénico

Bloqueo interescalénico, supraescapular, axilar, supraclavicular

Bloqueo supraclavicular, infraclavicular, axilar

Regional endovenosa, bloqueo de plexo braquial, bloqueo de nervios mediano, radial, cubital a nivel del codo.

Bloqueo de plexo lumbar, Bloqueo Cuadrado lumbar, ESP

Bloqueo femoral, Canal aductor, IPACK

Bloqueo femoral, Canal aductor, IPACK

Bloqueo ciático popliteo, bloqueo de tobillo

BPV: Bloqueo paravertebral, PECS: Bloqueo pectoral, ESP: Erector de la espina, TAP: Bloqueo transverso abdominal, IPACK: Infiltración entre la arteria popliteal y la capsula de 